\title{
Risk factors for selection of patients at high risk of recurrence or death after complete surgical resection in stage I gastric cancer
}

\author{
Ji Hyun Park • Min-Hee Ryu $\cdot$ Hwa Jung Kim • Baek-Yeol Ryoo • \\ Changhoon Yoo $\cdot$ Inkeun Park $\cdot$ Young Soo Park $\cdot$ Sung Tae Oh • \\ Jeong Hwan Yook • Byung Sik Kim • Yoon-Koo Kang
}

Received: 24 July 2014 / Accepted: 6 January 2015 / Published online: 23 January 2015

(c) The International Gastric Cancer Association and The Japanese Gastric Cancer Association 2015

\begin{abstract}
Background The therapeutic benefit of adjuvant chemotherapy has not been proven in stage I gastric cancer (GC). The aim of this study was to identify stage I GC patients at high risk of recurrence or death.

Methods We retrospectively reviewed the medical records of 2,783 patients with pathologically confirmed stage I GC who underwent curative surgical resection alone at Asan Medical Center between 2003 and 2007. The clinicopathologic parameters explored included age, sex, histologic differentiation, Lauren classification, size,
\end{abstract}

J.H. Park and M.-H. Ryu contributed equally and should be considered as first coauthors.

Electronic supplementary material The online version of this article (doi:10.1007/s10120-015-0464-5) contains supplementary material, which is available to authorized users.

J. H. Park · M.-H. Ryu · B.-Y. Ryoo · C. Yoo · I. Park ·

Y.-K. Kang $(\bowtie)$

Department of Oncology, Asan Medical Center,

University of Ulsan College of Medicine, 88 Olympic-ro 43-gil,

Songpa-gu, 138-736 Seoul, Korea

e-mail: ykkang@amc.seoul.kr

H. J. Kim

Department of Clinical Epidemiology and Biostatistics, Asan Medical Center, University of Ulsan College of Medicine, 88 Olympic-ro 43-gil, Songpa-gu, 138-736 Seoul, Korea

Y. S. Park

Department of Pathology, Asan Medical Center,

University of Ulsan College of Medicine, 88 Olympic-ro 43-gil,

Songpa-gu, 138-736 Seoul, Korea

S. T. Oh · J. H. Yook - B. S. Kim

Department of Surgery, Asan Medical Center,

University of Ulsan College of Medicine, 88 Olympic-ro 43-gil,

Songpa-gu, 138-736 Seoul, Korea location, multiplicity, stage, lymphovascular or perineural invasion, preoperative serum levels of tumor markers (carcinoembryonic antigen, carbohydrate antigen 19-9, carbohydrate antigen 72-4), and type of surgery.

Results With a median follow-up of 54 months (range 0-60 months), 212 patients ( $7.6 \%$ ) experienced recurrence or death, and the 5 -year recurrence-free survival (RFS) rate and overall survival rate were 89.9 and $93.4 \%$, respectively. With a multivariate analysis, six factors (age over 65 years, male gender, stage IB GC, lymphovascular invasion, perineural invasion, and elevated level of carcinoembryonic antigen) were independent poor prognostic factors for RFS $(p<0.05)$. Patients with more than two of six poor risk factors had a 5-year RFS rate of $79 \%$, whereas patients with fewer risk factors had a 5-year RFS rate of $97 \%(p<0.001)$.

Conclusions In this study cohort, we identified six independent risk factors for RFS. The patients with more than two risk factors are expected to have significant risk of recurrence or death after curative resection and should be considered as candidates for adjuvant treatment.

Keywords Stage I - Gastric cancer · Risk factor · Recurrence

\section{Introduction}

Gastric cancer (GC) is the fifth commonest malignancy and the third leading cause of cancer-related death worldwide, and it has the highest incidence and mortality rates in East Asia, including Japan and Korea [1-4]. In locally advanced cases, the only current available treatment for cure is complete surgical resection, and D2 resection is considered as the standard gastrectomy not only in East Asia but also 
in Western countries [2, 5-7]. In recent large clinical trials, including the Adjuvant Chemotherapy Trial of TS-1 for Gastric Cancer (ACTS-GC) and the Capecitabine and Oxaliplatin Adjuvant Study in Stomach Cancer (CLASSIC), the therapeutic benefit of adjuvant chemotherapy was established for patients with stage II and stage III GC [811]. As a result, adjuvant chemotherapy with $\mathrm{S}-1$ or a combination capecitabine and oxaliplatin regimen has become the standard treatment after D2 resection for stage II and stage III GC.

In Korea and Japan, the rate of detection of early-stage GC has been markedly increased in the last couple of decades because of nationwide screening programs with endoscopy. As a result, stage I GC currently accounts for approximately $50 \%$ of the total number of operations performed for GC in Japan and Korea [12-14]. Patients with early-stage GC generally have an excellent prognosis after curative (R0) resection. In cases of stage I GC, the 5and 10-year survival rates have been reported to be over $90 \%$ and $85-90 \%$, respectively $[15,16]$. However, there is recurrence in a small but definite number of patients even with stage I GC after curative resection. The recurrence rates for stage I GC after gastrectomy have been reported to range from 1.4 to $13.8 \%$ [15, 17-19]. Although several previous studies attempted to evaluate the risk factors for recurrence of or death from early-stage GC, there has been no common prognostic model established based on significant factors for stage I GC because of its relatively low incidence of recurrence or death. Moreover, unlike stage II and stage III GC, there is no global agreement on adjuvant chemotherapy for stage I GC because of a lack of randomized clinical trials of adjuvant treatment with sufficient statistical power.

Considering the expansion of the population with stage I GC and consequently the number of patients who would experience recurrence, it is necessary to identify patients with stage I GC at a high risk of recurrence or death who might receive therapeutic benefit from adjuvant chemotherapy. Hence, we performed a retrospective study to identify significant risk factors for recurrence or death for patients with stage I GC at high risk who might be potential candidates for future adjuvant trials.

\section{Patients and methods}

Patients and clinicopathologic parameters

Patients with newly diagnosed pathologically proven stage I GC according to the sixth edition of the American Joint Committee on Cancer (AJCC) staging system who underwent curative surgical resection from 2003 to 2007 at Asan
Medical Center, Seoul, Korea, were retrospectively reviewed. Among the patients reviewed, we excluded patients with initially advanced-stage GC but downstaged as stage I GC after neoadjuvant chemotherapy followed by curative resection, endoscopic mucosal resection, or endoscopic submucosal dissection as the initial surgical intervention, or initial curative resection at other hospitals. Patients with a history or concurrence of malignancies other than gastric adenocarcinoma were also excluded, except for patients fully recovered from nonmelanoma skin cancer and cervical carcinoma in situ. The clinical parameters of interest for recurrence are listed in Table 1. After surgical resection, typical follow-up workups to detect recurrences included physical examination every 3 months for 2 years and then every 6 months for the next 3 years, chest X-rays and abdominal and pelvic CT scans every 6 months, and gastroscopy every year for 5 years. Recurrences were classified into locoregional alone, distant alone, or both locoregional and distant relapses. This study was approved by the Institutional Review Board of Asan Medical Center.

\section{Statistical analysis}

The efficacy outcomes primarily included 5-year recurrence-free survival (RFS) and 5-year overall survival (OS) within 5 years following surgical resection. RFS was defined as the time from the date of curative resection to the time of confirmed recurrence of GC or death from any cause that occurred in the first 5 years, and OS was defined as the time from the curative resection to the time of death from any cause within 5 years. Patients were censored at 5 years for RFS and OS if they were recurrence free and alive at 5 years from the initial curative resection. In the case of patients who were lost to followup without evidence of recurrence but who were still alive at 5 years, they were censored at the date of the last clinic visit in RFS but were censored at the 5-year point for OS. The univariate and multivariate analyses of risk factors were performed using the Kaplan-Meier method with the log-rank test and the Cox proportional hazards model, respectively. The Cox proportional hazards model was used to calculate hazard ratios with variable selection procedures using the bootstrapping method. Finally, we constructed a provisional prognostic model to stratify the risk of RFS based on the risk factors that remained significant after multivariate analyses. All $p$ values were twosided, and a $p<0.05$ was considered statistically significant. The confidence intervals were determined at the $95 \%$ level. Statistical Package for the Social Sciences (version 20.0; SPSS, Chicago, IL, USA) was used for all statistical analyses. 
Table 1 Baseline patient characteristics before surgery
$A C$ adenocarcinoma, $C A$ carbohydrate antigen, $C E A$ carcinoembryonic antigen, $G E$ gastroesophageal, $L N$ lymph node, $S R C$ signet ring cell

${ }^{a}$ Median 58 years, range $19-89$ years

\begin{tabular}{|c|c|c|c|c|c|}
\hline Patient characteristics & No. & Percentage & Patient characteristics & No. & Percentage \\
\hline Age $(\text { years })^{\mathrm{a}}$ & & & $\mathrm{T}$ stage & & \\
\hline Median (range) & 58 & $19-89$ & Mucosa/submucosa (T1) & 2,146 & 77.1 \\
\hline$<65$ & 2,028 & 72.9 & Proper muscle (T2a) & 1,489 & 17.6 \\
\hline$\geq 65$ & 755 & 27.1 & Subserosa $(\mathrm{T} 2 \mathrm{~b})$ & 147 & 5.3 \\
\hline \multicolumn{6}{|l|}{ Sex } \\
\hline Male & 1,858 & 66.7 & $\mathrm{~N}$ stage & & \\
\hline Female & 926 & 33.3 & No & 2,665 & 95.9 \\
\hline Differentiation & & & N1 & 118 & 4.1 \\
\hline Good or moderate & 1,462 & 52.5 & Stage & & \\
\hline Poor & 1,290 & 46.3 & IA & 2,250 & 80.8 \\
\hline Unknown & 32 & 1.1 & IB & 533 & 19.2 \\
\hline WHO classification & & & Lymphovascular invasion & & \\
\hline Tubular AC & 2,238 & 80.4 & No & 2,550 & 91.6 \\
\hline SRC/mucinous & 518 & 18.6 & Yes & 232 & 8.3 \\
\hline Unknown & 27 & 1.0 & Unknown & 2 & 0.1 \\
\hline Lauren classification & & & Perineural invasion & & \\
\hline Intestinal & 1,537 & 55.2 & No & 2,348 & 84.3 \\
\hline Diffuse or mixed & 1,210 & 43.5 & Yes & 113 & 4.1 \\
\hline Unknown & 37 & 1.3 & Unknown & 323 & 11.6 \\
\hline Tumor size $(\mathrm{cm})$ & & & CEA level & & \\
\hline$<5$ & 2,281 & 81.9 & Normal & 2,609 & 93.1 \\
\hline$\geq 5$ & 499 & 17.9 & Above normal & 46 & 1.7 \\
\hline Tumor location & & & Unknown & 132 & 4.7 \\
\hline Angle or antrum & 1,489 & 53.5 & CA 19-9 level & & \\
\hline Body or fundus & 1,052 & 37.8 & Normal & 2,590 & 93.1 \\
\hline Cardia or GE junction & 123 & 4.4 & Above normal & 46 & 1.7 \\
\hline Multiple & 103 & 3.7 & Unknown & 147 & 5.3 \\
\hline Unknown & 17 & 0.6 & CA 72-4 level & & \\
\hline Multiplicity & & & Normal & 2,329 & 83.7 \\
\hline No & 2,681 & 96.3 & Above normal & 203 & 7.3 \\
\hline Yes & 103 & 3.7 & Unknown & 251 & 9.0 \\
\hline Type of surgery & & & Type of LN dissection & & \\
\hline Total gastrectomy & 56 & 20.1 & D1 & 10 & 0.5 \\
\hline Distal gastrectomy & 221 & 79.5 & D1+ & 1,520 & 54.6 \\
\hline Other types of surgery & 11 & 0.4 & D2 & 1,253 & 45.0 \\
\hline Proximal gastrectomy & 3 & 0.1 & & & \\
\hline Wedge resection & 8 & 0.3 & & & \\
\hline
\end{tabular}

\section{Results}

Patients and clinicopathologic characteristics

From January 2003 to December 2007, stage I GC was newly diagnosed in 4,201 patients after curative resection at Asan Medical Center. Of these, 197 patients were excluded from the analysis for previously described reasons (for details, see Fig. 1). Among the remaining 3,904 patients, we additionally excluded 1,121 patients who received any type of adjuvant treatment, and we finally analyzed 2,783 patients with pathologically confirmed stage I GC who had not received adjuvant treatment after curative resection (Fig. 1). The clinicopathologic characteristics of the 2,783 patients are summarized in Table 1.

Overall outcomes and pattern and frequency of recurrence

The median follow-up period was 54 months (range 0-60 months). Among 2,783 patients, 84 patients (3.0\%) experienced recurrences, 179 patients $(6.4 \%)$ died, and 


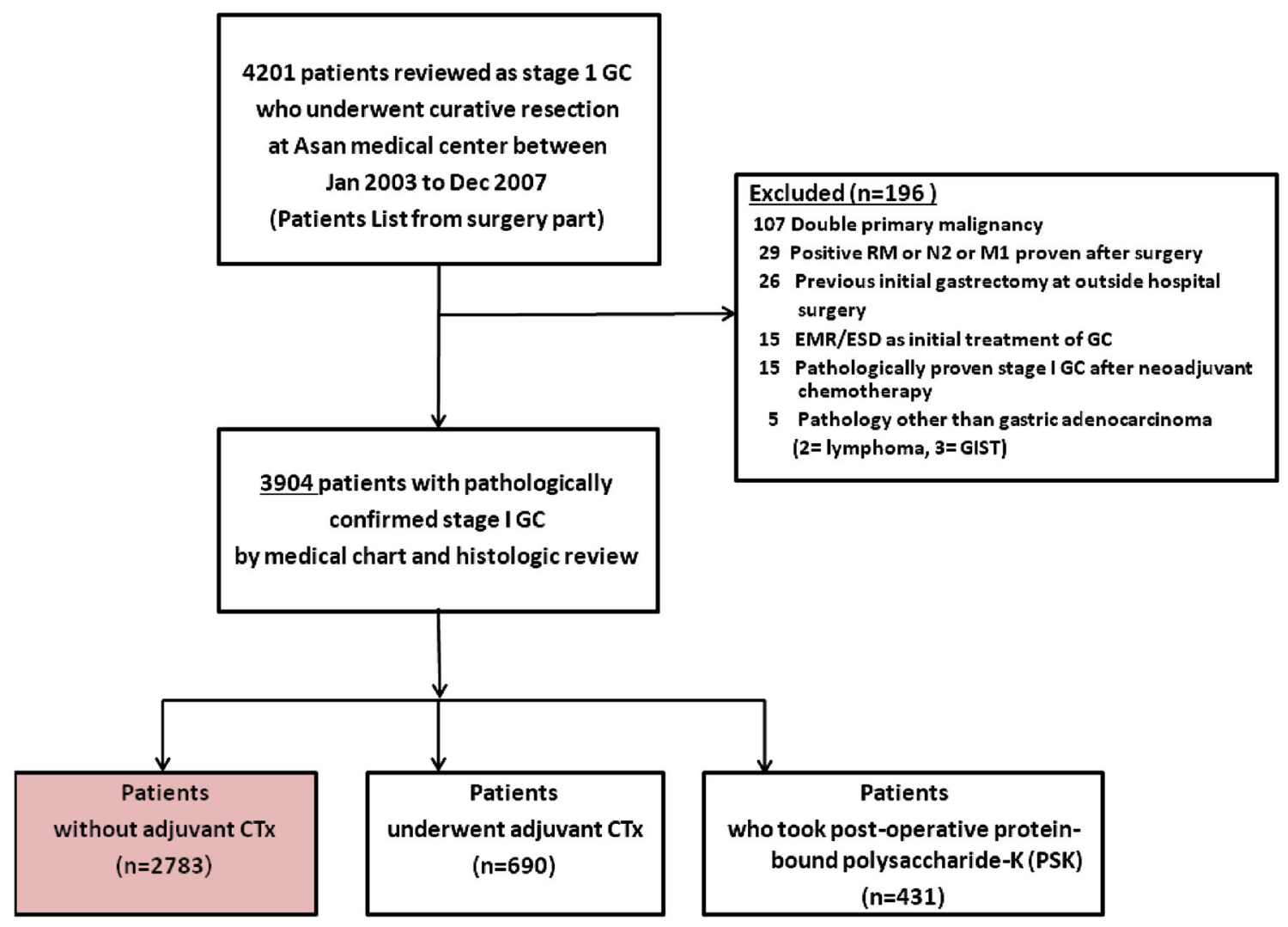

Fig. 1 Consolidated Standards of Reporting Trials (CONSORT) diagram of patients. CTx chemotherapy, EMR endoscopic mucosal resection, $E S D$ endoscopic submucosal dissection, $G C$ gastric cancer, GIST gastrointestinal stromal tumor, $R M$ resection margin

212 patients $(7.6 \%)$ experienced recurrence or death within the 5-year follow-up. In all patients, the 5-year RFS and OS estimated by the Kaplan-Meier method were 89.9 and $93.4 \%$, respectively (Fig. 2). Among 84 patients with recurrence, the median time to recurrence was 25.7 months (range 1.3-55.8 months), and 37 patients (44.0 \%) experienced relapse within 2 years. Among 84 patients with recurrence, 35 patients $(41.7 \%)$ displayed only locoregional relapse including the site of anastomosis, stomach, duodenum, and regional lymph nodes, 41 patients (48.8\%) had distant metastasis alone, and eight patients $(9.5 \%)$ had both distant and locoregional recurrence. Among 49 patients with distant metastasis, the peritoneum was the commonest metastatic site $(n=17,34.7 \%)$, followed by the liver $(n=15,30.6 \%)$, lung or pleura $(n=7,14.3 \%)$, extra-abdominal lymph nodes $(n=5,10.2 \%)$, bone $(n=4,8.2 \%)$, and brain $(n=1,2.0 \%)$.

\section{Analysis of risk factors for recurrence and death}

All clinicopathologic factors described earlier were sequentially evaluated by the Kaplan-Meier method and using a Cox proportional hazards model. The results are summarized in Table 2. In the univariate analyses, lower
Fig. 2 Survival outcomes of all patients $(n=2,783)$. OS overall survival, RFS recurrence-free survival
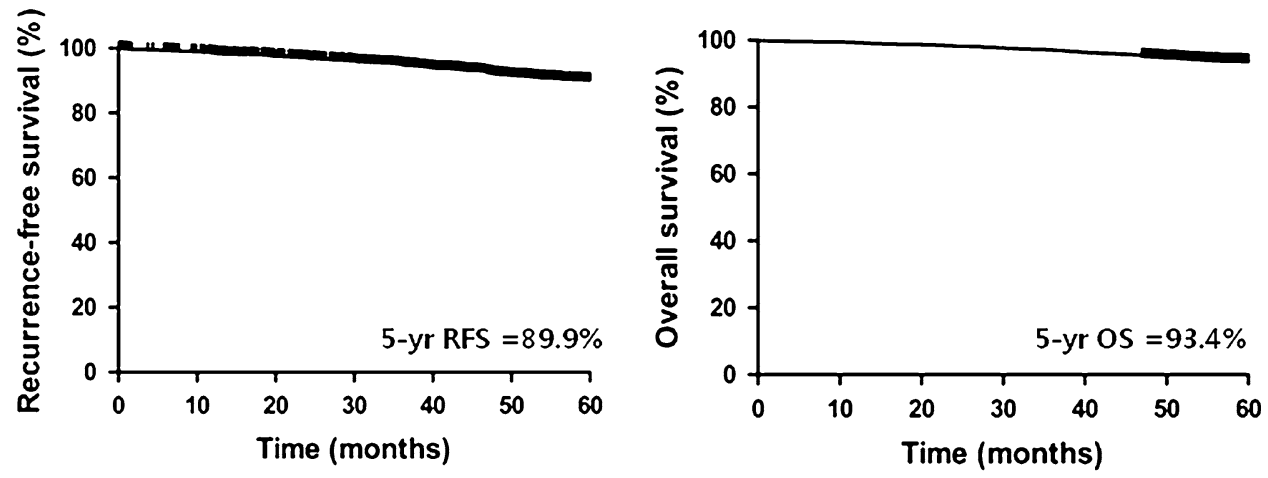
Table 2 Risk factors for recurrence-free survival

$C A$ carbohydrate antigen, $C E A$ carcinoembryonic antigen, $C I$ confidence interval, $H R$ hazard ratio

\begin{tabular}{llllr}
\hline Clinicopathologic factors & Univariate analysis & \multicolumn{3}{l}{ Multivariate analysis } \\
\cline { 4 - 5 } & $p$ & HR & $95 \%$ CI & $p$ value \\
\hline Age ( $\geq 65$ years vs $<65$ years) & $<0.001$ & 4.55 & $3.18-6.52$ & $<0.001$ \\
Sex (male vs female) & $<0.001$ & 1.60 & $0.45-0.86$ & 0.004 \\
Differentiation (poor vs good or moderate) & 0.001 & & & \\
Lauren classification (intestinal vs diffuse or mixed) & 0.039 & & & \\
Multiple tumors ( $\geq 2$ vs $<2$ ) & 0.041 & & & \\
T staging & $<0.001$ & & & \\
Mucosa or submucosa (T1) & & & & \\
Proper muscle (T2a) & & & & \\
Subserosa (T2b) & & 1.69 & $1.023-2.78$ \\
Lymphovascular invasion (yes vs no) & $<0.001$ & 1.87 & $1.16-3.00$ & 0.010 \\
Perineural invasion (yes vs no) & $<0.001$ & & & $<0.001$ \\
No. of involved lymph nodes (N1 vs N0) & $<0.001$ & 2.05 & $1.50-2.79$ & $<0.001$ \\
Stage (IB vs IA) & $<0.001$ & 1.83 & $1.05-2.16$ & \\
CEA level (above normal vs normal) & 0.003 & & & \\
CA 19-9 level (above normal vs normal) & $<0.001$ & &
\end{tabular}

5-year RFS was significantly associated with age above 65 years, male gender, poorly differentiated adenocarcinoma, intestinal type, multiple tumors, T2 and N1 stage, stage IB GC, lymphovascular and perineural invasion, and elevation of serum carcinoembryonic antigen (CEA) and carbohydrate antigen 19-9 levels, which similarly were predictive of a lower 5-year OS. In the multivariate analysis, we included both $\mathrm{T}$ and $\mathrm{N}$ stage separately in addition to overall stage to evaluate their relative prognostic impact. As a result, we finally identified six factors that remained significant for lower RFS (age over 65 years, male gender, stage IB GC, lymphovascular invasion, perineural invasion, and elevated serum level of CEA) $(p<0.05)$. Four of the factors, not including the presence of lymphovascular and perineural invasion, were also significantly related to lower OS $(p<0.05)$.

Patient selection by risk factor analysis

A risk factor analysis was performed to classify patients into different risk groups and select the patients with higher risk. A total of 2,338 out of 2,783 patients were recruited in the analysis because of the documented missing values of covariates. We assigned the same score of one point to each of six variables to equate the scores to the number of risk factors. The range of the sum of the scores was from zero to five, and the RFS rates of each score group were 98.5, 98.1, 83.8, 80.1, 71.8, and $80.0 \%$ at 5 years, respectively $(p<0.001)$. On the basis of these results, two risk groups were identified according to the number of risk factors. Patients with more than two risk factors were defined as the high-risk group, and the all others were defined as the low-risk group. The numbers of patients in the low-risk and high-risk groups were 2,136 (91.4\%) and $202(8.6 \%)$, respectively. In survival analysis, patients with three or more risk factors had a significantly lower 5-year RFS rate of $72.3 \%$, whereas patients with one or two risk factors had a 5-year RFS rate of $91.8 \%$ $(p<0.001)$. The high-risk-group and low-risk-group 5-year OS rates were 83.4 and $95.0 \%$, respectively (Fig. 3).

\section{Discussion}

According to a recent report, the proportion of patients with early-stage GC in Korea increased from $28.6 \%$ in 1995 to $57.7 \%$ in 2009, whereas the overall incidence of GC did not change during the same period $[12,13]$. Although the prognosis of stage I GC is known to be excellent in general after curative surgery and we also observed an RFS rate of approximately $90 \%$ at 5 years in our series, postoperative recurrences and/or deaths still occur in a certain percentage of patients with various relapse rates [15, 17-19]. With more detection of stage I GC in Korea and Japan, the absolute number of patients with stage I GC who experience recurrence is also increasing, which may result in a large health problem in those countries. Given the absence of recurrence in most patients with stage I GC, determining the parameters for a high risk of recurrence, which was addressed in this study, is a prerequisite for selecting candidates for adjuvant treatment.

In the current study, we demonstrated that several clinicopathologic parameters are significantly related to a 


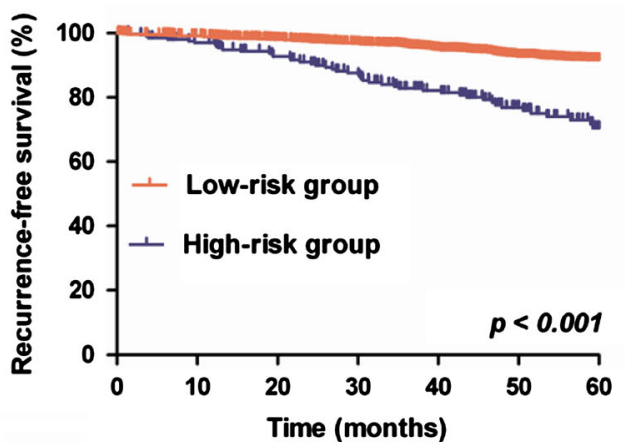

$\begin{array}{lrrrrrrr}\begin{array}{l}\text { Number of patients at risk \& } \\ \text { cumulated events }\end{array} & & & & & & \\ \text { Risk factors }<3 & 2136 & 2009 & 1885 & 1780 & 1559 & 1160 & 695 \\ & & 6 & 14 & 22 & 29 & 35 & 42 \\ \text { Risk factors } \geq 3 & 202 & 177 & 158 & 141 & 112 & 81 & 49 \\ & & 13 & 33 & 52 & 79 & 109 & 127\end{array}$

Fig. 3 Recurrence-free survival (RFS) and overall survival according to the number of independent risk factors identified from multivariate analysis. Patients in the low-risk group have one or two risk factors, and patients in the high-risk group have three or more risk factors. Risk factors for RFS include age over 65 years, male gender, stage IB

high risk of recurrence and/or death. Our study has salient strength in that we have analyzed the largest dataset of stage I GC with sufficient events thus far. Our analyses revealed six final independent risk factors for RFS by multivariate analysis, which were age over 65 years, male gender, stage IB GC, lymphovascular invasion, perineural invasion, and elevated serum level of CEA. Although the overall stage is determined by a combination of $\mathrm{T}$ and $\mathrm{N}$ stage, our findings may suggest that the overall stage may independently affect the prognosis beyond each of $\mathrm{T}$ stage and $\mathrm{N}$ stage. Of the six independent variables we observed, one or more were repeatedly observed as risk factors for recurrence in each of the previous studies with a smaller patient population with early-stage GC [12, 15-29]. In general, metastasis to lymph nodes has been reported to be one of the most significant risk factors for recurrence of early-stage GC [17, 29]. The depth of invasion is generally related to metastasis to lymph nodes [18, 29]; lymphatic or vascular invasion, submucosal invasion, and old age are other risk factors for metastasis to lymph nodes. In a few studies including only stage IB GC, undifferentiated adenocarcinoma, tumor size greater than $3 \mathrm{~cm}$, and the presence of perineural invasion were also observed as poor prognostic factors $[26,28]$. Notably, distinct from previous studies, we included serum levels of tumor markers as relevant clinicopathologic factors, and multivariate analysis revealed CEA as an independent predictor for RFS. Our study suggests that tumor markers might also be considered for risk stratification in future clinical trials of adjuvant chemotherapy in early-stage GC.

By grouping the patients on the basis of six independent risk factors, we illustrated two risk groups with distinct

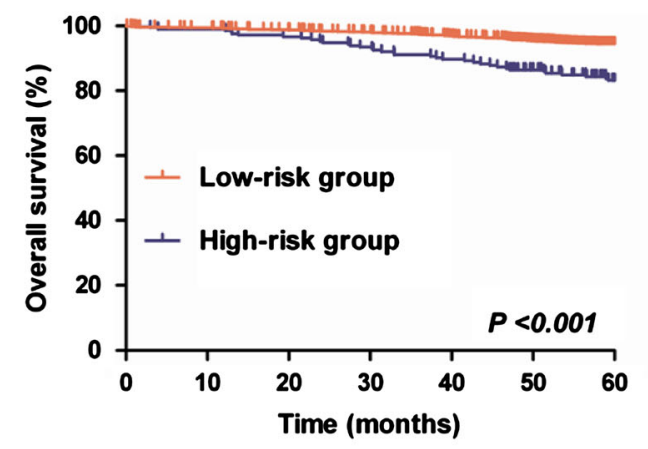

Number of patients at risk \& cumulatedevents

$\begin{array}{llllllll}\text { Risk factors }<3 & 2136 & 2130 & 2120 & 2105 & 2087 & 1980 & 1727\end{array}$

$\begin{array}{lrrrrrrr}\text { Risk factors } \geq 3 & 202 & 199 & 195 & 189 & 181 & 164 & 128 \\ & & 7 & 17 & 32 & 50 & 78 & 104\end{array}$

gastric cancer according to the sixth edition of the American Joint Committee on Cancer staging system, lymphovascular invasion, perineural invasion, and elevated serum level of carcinoembryonic antigen

survival outcomes, i.e., a high-risk group with a 5-year RFS rate of $72.3 \%$ and a low-risk group with a 5-year RFS rate of $91.8 \%$. Because of a lack of clinical trials with sufficient statistical power, adjuvant treatment is generally not recommended for patients with stage I GC. Thus far, large-scale clinical trials of adjuvant treatment in GC have mainly included patients with stage II and stage III GC but no or too few patients with stage I GC $[8-11,30,31]$. According to ACTS-GC, which compared adjuvant S-1 with surgery alone after curative resection in stage II or stage III GC, the 5-year RFS rate was better with adjuvant S-1 compared with surgery alone $(79.2 \%$ vs $64.4 \%$, respectively) in patients with stage II GC [30]. Considering that patients with a less advanced stage of GC benefited more from adjuvant chemotherapy than patients with a more advanced stage (hazard ratios for RFS of 0.521 in stage II GC, 0.696 in stage IIIA GC, and 0.788 in stage IIIB $\mathrm{GC}$ ), and the 5-year RFS rate of the high-risk group identified in our study is close to that of the stage II GC group in ACTS-GC, it is expected that stage I GC patients belonging to the high-risk group might benefit from adjuvant chemotherapy after curative resection. Kunisaki et al. [16] also observed some patients with stage I GC whose long-term outcomes are as poor as those of patients with stage II GC. They studied 1,880 patients with histologically proven stage I GC to identify lymphovascular invasion as a poor prognostic factor in stage IB GC patients. In their report, patients with $\mathrm{T} 2 \mathrm{~N} 0 \mathrm{GC}$ having lymphovascular invasion and stage II GC had similar survival outcomes, which suggests T2N0 patients with moderate to severe lymphovascular invasion are suitable for adjuvant chemotherapy. In addition, a more recent study demonstrated a 
prognostic scoring system based on three risk factorstumor diameter, lymphovascular invasion, and perineural invasion in pT2N0 GC patients-which categorized patients into three prognostically different risk groups [26]. Their results also suggest adjuvant treatment is required for some patients with stage I GC.

In the current study, we declared the extent of lymph node dissection (LND) applied in curative resection of GC, which revealed $54.6 \% \mathrm{D} 1+$ dissection in addition to $45.0 \%$ D2 dissection. Although the principle of gastric resection and LND in GC generally follows the Japanese GC treatment guidelines [32, 33] in our institute, which means surgeons determin the extent of LND on the basis of the clinical stage evaluated prior to gastrectomy using CT or endoscopic examination, some exceptions were made in unusual circumstances as elderly patients, major comorbidities including heart and liver problems, or surgeryrelated complications. There were no significant differences in RFS between D1+ and D2 dissection in either all the patients or patients with stage IB GC (Fig. S1). Considering that there is no established guideline or prospective study comparing D1+ and D2 dissection in entire stage I GC patients, further randomized controlled trials are warranted.

This study also has some limitations. The first is that we used the sixth edition of the AJCC staging system rather than current, seventh edition. However, considering all of the recently published trials of adjuvant therapy in GC [8, 30] explored the efficacy of adjuvant chemotherapy on the basis of the sixth edition of the AJCC staging system, and our study aimed to find potential candidates for adjuvant chemotherapy among stage I GC patients, it seemed reasonable to perform this study on the basis of the sixth edition of the AJCC staging system to better compare the efficacy outcomes with those of other reference studies of adjuvant chemotherapy. Second, the current study does not provide data analysis in patients treated with endoscopic mucosal resection or endoscopic submucosal dissection, which is an increasingly frequent procedure in Korea and Japan. We excluded patients who had undergone only endoscopic resection because adjuvant treatment is usually not recommended after endoscopic resection in principle, and accordingly they were not considered as candidates for adjuvant chemotherapy. Moreover, the exact status of lymph node involvement was unknown with endoscopic mucosal resection or endoscopic submucosal dissection, but we needed information on involvement of lymph nodes with surgery to figure out the risk factors for relapse or death. Third, even though most patients with stage I GC do not experience recurrence or death within 5 years, a substantial number of cases were censored for RFS, which might lessen the power of the study. However, in stage I GC unlike in advanced stages of GC, particularly in cases without recurrence, patients are often not willing to continue having regular checkups after curative resection because of its excellent prognosis in general. Among patients lost to follow-up within 2 years after surgery in our study, most patients $(94.5 \%)$ were alive at 5 years. Thus, censored cases from follow-up loss, on one hand, may reflect real-world practice and may be more prominent in a retrospective study. Moreover, the current study achieved statistical significance in spite of many censored cases. Lastly, we did not perform external or internal validation of our risk model. However, it might be difficult to construct a dataset including a large patient population such as ours for external validation. And despite the large cohort size in this study, the number of events was considered not big enough to divide patients into training and validation sets for internal validation.

In summary, in our large cohort of patients with stage I GC irrespective of their $\mathrm{T}$ or $\mathrm{N}$ stages, age over 65 years, male gender, stage IB GC, lymphovascular invasion, perineural invasion, and elevated serum level of CEA were six independent risk factors for recurrence or death. Furthermore, risk factor analysis was performed for risk stratification by which stage I GC patients could be categorized into two different risk groups displaying a 5-year RFS rate difference near 20 percentage points. Taken together, our results suggest that there are stage I GC patients with a higher risk of recurrence and/or death who may proceed to additional adjuvant chemotherapy after surgical resection. Considering the large number of patients for whom there is comprehensive information on the clinicopathologic parameters in this study, our results could be generalized, at least in East Asia, where the same surgical procedures are routinely practiced. On the basis of the results of this study, a prospective randomized clinical trial of adjuvant chemotherapy versus surgery alone in stage I GC patients at high risk of recurrence or death is now ongoing (ClinicalTrials.gov identifier NCT01917552).

Acknowledgments This work was supported by a grant from the National R\&D Program for Cancer Control, Ministry of Health and Welfare, Republic of Korea (1420240).

Conflict of interest The authors declare they have no conflict of interest

\section{References}

1. Jemal A, Siegel R, Xu J, Ward E. Cancer statistics, 2010. CA Cancer J Clin. 2010;60:277-300.

2. Miyahara R, Niwa Y, Matsuura T, et al. Prevalence and prognosis of gastric cancer detected by screening in a large Japanese population: data from a single institute over 30 years. J Gastroenterol Hepatol. 2007;22:1435-42.

3. Kamangar F, Dores GM, Anderson WF. Patterns of cancer incidence, mortality, and prevalence across five continents: defining 
priorities to reduce cancer disparities in different geographic regions of the world. J Clin Oncol. 2006;24:2137-50.

4. Ferlay J SI, Ervik M, Dikshit R, Eser S, Mathers C, Rebelo M, Parkin DM, Forman D, Bray, F. GLOBOCAN 2012 v1.0, Cancer Incidence and Mortality and Worldwide: IARC CancerBase no. 11. Lyon: International Agency for Research on Cancer. 2013.

5. Desai AM, Pareek M, Nightingale PG, Fielding JW. Improving outcomes in gastric cancer over 20 years. Gastric Cancer. 2004;7:196-201; discussion 201-3.

6. Songun I, Putter H, Kranenbarg EM, et al. Surgical treatment of gastric cancer: 15-year follow-up results of the randomised nationwide Dutch D1D2 trial. Lancet Oncol. 2010;11:439-49.

7. Sano T, Sasako M, Yamamoto S, et al. Gastric cancer surgery: morbidity and mortality results from a prospective randomized controlled trial comparing D2 and extended para-aortic lymphadenectomy_Japan Clinical Oncology Group Study 9501. J Clin Oncol. 2004;22:2767-73.

8. Bang YJ, Kim YW, Yang HK, et al. Adjuvant capecitabine and oxaliplatin for gastric cancer after D2 gastrectomy (CLASSIC): a phase 3 open-label, randomised controlled trial. Lancet. 2012;379:315-21.

9. Sakuramoto S, Sasako M, Yamaguchi T, et al. Adjuvant chemotherapy for gastric cancer with S-1, an oral fluoropyrimidine. N Engl J Med. 2007;357:1810-20.

10. Macdonald JS, Smalley SR, Benedetti J, et al. Chemoradiotherapy after surgery compared with surgery alone for adenocarcinoma of the stomach or gastroesophageal junction. N Engl J Med. 2001;345:725-30.

11. Cunningham D, Allum WH, Stenning SP, et al. Perioperative chemotherapy versus surgery alone for resectable gastroesophageal cancer. N Engl J Med. 2006;355:11-20.

12. Kim JW, Hwang I, Kim MJ, Jang SJ. Clinicopathological characteristics and predictive markers of early gastric cancer with recurrence. J Korean Med Sci. 2009;24:1158-64.

13. Jeong O, Park YK. Clinicopathological features and surgical treatment of gastric cancer in South Korea: the results of 2009 nationwide survey on surgically treated gastric cancer patients. J Gastric Cancer. 2011;11:69-77.

14. Park JM, Kim YH. Current approaches to gastric cancer in Korea. Gastrointest Cancer Res. 2008;2:137-44.

15. Sano T, Sasako M, Kinoshita T, Maruyama K. Recurrence of early gastric cancer. Follow-up of 1475 patients and review of the Japanese literature. Cancer. 1993;72:3174-8.

16. Kunisaki C, Makino H, Kimura J, et al. Impact of lymphovascular invasion in patients with stage I gastric cancer. Surgery. 2010;147:204-11.

17. Kunisaki C, Shimada H, Takahashi M, et al. Prognostic factors in early gastric cancer. Hepatogastroenterology. 2001;48:294-8.

18. Lee HJ, Kim YH, Kim WH, et al. Clinicopathological analysis for recurrence of early gastric cancer. Jpn J Clin Oncol. 2003;33:209-14.
19. Youn HG, An JY, Choi MG, et al. Recurrence after curative resection of early gastric cancer. Ann Surg Oncol. 2010;17:448-54.

20. Habu H, Takeshita K, Sunagawa M, Endo M. Prognostic factors of early gastric cancer-results of long-term follow-up and analysis of recurrent cases. Jpn J Surg. 1987;17:248-55.

21. Folli S, Dente M, Dell'Amore D, et al. Early gastric cancer: prognostic factors in 223 patients. Br J Surg. 1995;82:952-6.

22. Wu CY, Chen JT, Chen GH, Yeh HZ. Lymph node metastasis in early gastric cancer: a clinicopathological analysis. Hepatogastroenterology. 2002;49:1465-8.

23. Komatsu S, Ichikawa D, Kurioka H, et al. Prognostic and clinical evaluation of patients with T2 gastric cancer. Hepatogastroenterology. 2005;52:965-8.

24. Saito H, Osaki T, Murakami D, et al. Recurrence in early gastric cancer-presence of micrometastasis in lymph node of node negative early gastric cancer patient with recurrence. Hepatogastroenterology. 2007;54:620-4.

25. Lai JF, Kim S, Kim K, et al. Prediction of recurrence of early gastric cancer after curative resection. Ann Surg Oncol. 2009;16:1896-902.

26. Du C, Zhou Y, Huang K, et al. Defining a high-risk subgroup of pathological T2N0 gastric cancer by prognostic risk stratification for adjuvant therapy. J Gastrointest Surg. 2011;15:2153-8.

27. Kim BS, Cho SW, Min SK, Lee BH. Differences in prognostic factors between early and advanced gastric cancer. Hepatogastroenterology. 2011;58:1032-40.

28. Yokoyama T, Kamada K, Tsurui Y, et al. Clinicopathological analysis for recurrence of stage Ib gastric cancer (according to the second English edition of the Japanese classification of gastric carcinoma). Gastric Cancer. 2011;14:372-7.

29. Gunji Y, Suzuki T, Hori S, et al. Prognostic significance of the number of metastatic lymph nodes in early gastric cancer. Dig Surg. 2003;20:148-53.

30. Sasako M, Sakuramoto S, Katai H, et al. Five-year outcomes of a randomized phase III trial comparing adjuvant chemotherapy with $\mathrm{S}-1$ versus surgery alone in stage II or III gastric cancer. J Clin Oncol. 2011;29:4387-93.

31. Nakajima T, Nashimoto A, Kitamura M, et al. Adjuvant mitomycin and fluorouracil followed by oral uracil plus tegafur in serosa-negative gastric cancer: a randomised trial. Gastric cancer surgical study group. Lancet. 1999;354:273-7.

32. Japanese Gastric Cancer Association. Japanese gastric cancer treatment guidelines 2010 (ver. 3). Gastric Cancer. 2011;14(2):113-23.

33. Japanese Gastric Cancer Association. Japanese classification of gastric carcinoma: 3rd English edition. Gastric Cancer. 2011;14:101-12. 